\title{
COMPARAÇÃO DA LABILIDADE DE METAIS EMPREGANDO VOLTAMETRIA, DIFUSÃO EM FILMES FINOS POR GRADIENTE DE CONCENTRAÇÃO (DGT) E MODELO COMPUTACIONAL
}

\author{
Vanessa E. dos Anjos, Gilberto Abate e Marco T. Grassi* \\ Departamento de Química, Universidade Federal do Paraná, CP 19081, 81531-990 Curitiba - PR, Brasil
}

Recebido em 1/10/09; aceito em 4/2/10; publicado na web em 18/6/10

\begin{abstract}
COMPARISON OF THE SPECIATION OF TRACE METALS IN FRESHWATER EMPLOYING VOLTAMMETRY, DIFFUSIVE GRADIENTS IN THIN FILMS (DGT) AND A CHEMICAL EQUILIBRIUM MODEL. Speciation of metals in a synthetic freshwater was comparatively evaluated using Anodic Stripping Voltammetry, Diffusive Gradients in Thin Films and a Chemical Equilibrium Model. The labile fractions of $\mathrm{Cu}$ and Zn quantified by DGT were similar to the ones measured by ASV. The labile species of Cd and $\mathrm{Pb}$ could not be determined by both experimental methods due to the formation of inert complexes with organic ligands in the sample. Despite the differences among the methods, the speciation results obtained by the use of DGT and ASV agreed well with predictions made by the chemical equilibrium model.
\end{abstract}

Keywords: trace metals; speciation; lability.

\section{INTRODUÇÃo}

Em ambientes aquáticos naturais, metais traço são encontrados em uma variedade de formas físico-químicas e espécies, que governam sua mobilidade, biodisponibilidade e toxicidade frente a organismos aquáticos. ${ }^{1}$ Esses elementos estão presentes associados ao material particulado em suspensão e na fração dissolvida na forma de íons livres hidratados e complexados a ligantes orgânicos e inorgânicos. ${ }^{2}$ Porém, nem todas essas espécies são biodisponíveis, dado que a resposta biológica dos organismos está relacionada com a atividade das espécies metálicas lábeis, que são os íons livres hidratados e aqueles metais fracamente complexados. ${ }^{1}$

Dessa maneira, o conhecimento das formas que uma espécie metálica pode assumir no ambiente, ou seja, sua especiação, representa aspecto chave na compreensão de seus ciclos biogeoquímicos e de seus efeitos ecotoxicológicos. ${ }^{3}$

Um grande esforço tem sido devotado ao desenvolvimento e aprimoramento de ferramentas analíticas para a análise de especiação de metais. Idealmente, tais protocolos devem ser simples, de baixo custo, robustos e, ainda, devem possibilitar a compreensão do comportamento dinâmico das espécies metálicas, principalmente sobre a labilidade, aspecto crucial em estudos ecotoxicológicos. ${ }^{4}$ Inúmeros protocolos analíticos vêm sendo propostos, baseados em diferentes abordagens experimentais e conceitos, sendo capazes de detectar espécies metálicas de acordo com propriedades tais como tamanho, mobilidade, estabilidade e labilidade. ${ }^{4,5}$

As abordagens experimentais e modelagens mais clássicas utilizadas na análise de especiação de metais são baseadas em princípios de equilíbrio químico, pois se considera que o sistema aquático se encontra em equilíbrio. ${ }^{4}$ Nas abordagens experimentais são empregados procedimentos ex situ, ou seja, que envolvem etapas de amostragem, transporte, armazenamento, tratamento das amostras e detecção das espécies de interesse. Problemas associados a esses procedimentos são numerosos e estão relacionados com a possibilidade de adulteração das amostras, devido a contaminações ou perdas do analito pela instabilidade das espécies ou em decorrência da sua sorção às paredes dos frascos de armazenamento., ${ }^{4,6}$

A voltametria de redissolução anódica, ASV - anodic stripping voltammetry, é uma das ferramentas analíticas consideradas clássicas

*e-mail: mtgrassi@quimica.ufpr.br em estudos de especiação de metais. A ASV apresenta vantagens como baixo custo e elevada sensibilidade, além da possibilidade de avaliar a interação de íons metálicos com os ligantes presentes nas amostras. ${ }^{3,4,7} \mathrm{Na}$ ausência de tratamentos químicos da amostra, a resposta obtida na ASV deve-se unicamente a espécies metálicas definidas como lábeis, ou seja, o íon metálico livre e os complexos metálicos cineticamente reversíveis. ${ }^{4}$

Nos estudos de especiação também vêm sendo utilizados programas computacionais baseados em cálculos de equilíbrio químico. Diferentes modelos para a complexação de íons metálicos pelos ligantes comumente presentes em águas naturais são aplicados em tais programas. ${ }^{2,6}$ Assim, modelos computacionais podem ser considerados ferramentas úteis na previsão da especiação de espécies metálicas de acordo com as características químicas do ambiente avaliado. Vários programas estão disponíveis para o cálculo da concentração de íons metálicos presentes em um sistema definido. Porém, a obtenção de dados mais fidedignos acerca da especiação está condicionada ao conhecimento completo da composição da amostra, da concentração total de todos os seus componentes e, também, do conhecimento de todas as constantes de equilíbrio das reações que ocorrem em um dado sistema. ${ }^{4-6,8}$ Além disso, aspectos cinéticos não são considerados nos cálculos da distribuição do íon metálico entre as diferentes espécies e nos dias atuais é crescente o reconhecimento da importância de tais processos no controle do comportamento das espécies metálicas em águas naturais. ${ }^{2,5}$ Como ambientes aquáticos estão sujeitos a condições muito variáveis, o conhecimento de fatores dinâmicos torna-se importante em estudos envolvendo a mobilidade e a biodisponibilidade das espécies metálicas.

Nesse sentido, a análise de especiação dinâmica de metais vem surgindo como uma poderosa alternativa no entendimento de processos que não estão somente relacionados com a distribuição das espécies metálicas em equilíbrio, mas também com aspectos cinéticos e com a interconversão das espécies envolvidas. ${ }^{5}$ Geralmente, nesse tipo de abordagem são empregados dispositivos que possibilitam realizar medidas no próprio ambiente de interesse, minimizando os problemas de adulteração das amostras durante as etapas do processo analítico. ${ }^{9}$

Uma das ferramentas analíticas que visam à análise de especiação dinâmica de metais é a difusão em filmes finos por gradiente de concentração, DGT - diffusive gradients in thin films, desenvolvida por Davison e Zhang para ser utilizada na determinação in situ de 
espécies metálicas lábeis em águas naturais. ${ }^{9}$ As medidas baseiam-se na difusão de espécies metálicas através de um hidrogel, devido a um gradiente de concentração, com posterior acumulação das espécies lábeis em um agente ligante que é composto por uma resina quelante (Chelex-100 ${ }^{\circledR}$ ) imobilizada em um segundo hidrogel. Sob condições controladas os íons metálicos retidos na resina são eluídos com uma solução ácida e suas concentrações podem ser determinadas pela técnica analítica disponível. ${ }^{9}$

Os dispositivos DGT permitem discriminar as espécies metálicas que sofrem difusão no hidrogel de acordo com a labilidade, coeficientes de difusão e cinética de dissociação dos complexos metálicos durante o tempo da medida. ${ }^{10}$

Levando todos estes aspectos em consideração, é possível afirmar que estudos comparativos entre os protocolos analíticos disponíveis para a análise da especiação de metais tornam-se necessários, uma vez que os diversos protocolos estão fundamentados em diferentes conceitos. ${ }^{4}$ Por isso, estudos desenvolvidos sob condições rigorosamente controladas representam um pré-requisito na validação dos protocolos e no conhecimento das vantagens e limitações das diferentes abordagens. ${ }^{11}$

Embora existam na literatura estudos envolvendo o desempenho de diferentes protocolos na análise de especiação de metais para amostras de águas naturais e sintéticas, diversos autores relatam que medidas comparativas entre DGT e outros métodos ainda são necessárias. ${ }^{4,6,11}$

Neste contexto, o presente trabalho teve como objetivo realizar um estudo comparativo empregando ASV, DGT e um modelo computacional de especiação, na análise de especiação dos íons cobre, cádmio, chumbo e zinco. Na avaliação comparativa da labilidade das espécies metálicas foram conduzidos experimentos em um sistema modelo, sob condições controladas, empregando amostras sintéticas de água natural.

\section{PARTE EXPERIMENTAL}

Nos experimentos realizados foram adotados procedimentos de técnicas limpas visando à minimização de processos de contaminação e adulteração das amostras, conforme descrito por Campos e colaboradores. ${ }^{12}$ Todos os reagentes utilizados foram de grau analítico ou superior. As soluções dos íons metálicos foram preparadas a partir da diluição de soluções padrão de $1000 \mathrm{mg} \mathrm{L}^{-1}$ (Tritisol, Merck).

Com o emprego da ASV, DGT e modelo computacional realizouse a análise de especiação dos íons metálicos $\mathrm{Cu}^{2+}, \mathrm{Cd}^{2+}, \mathrm{Pb}^{2+} \mathrm{e} \mathrm{Zn}^{2+}$ no sistema modelo, a fim de proporcionar uma avaliação mais confiável em estudos comparativos. ${ }^{11}$ Uma amostra sintética $(8 \mathrm{~L})$ foi preparada contendo íons metálicos e complexantes com diversas constantes de estabilidade, sendo estes encontrados comumente em ambientes aquáticos. ${ }^{13}$ De modo análogo, o pH da amostra foi ajustado para 7,0 visando representar condições compatíveis com o meio aquático. A composição da amostra está apresentada na Tabela 1 .

A amostra foi agitada manualmente e ocasionalmente (mínimo de 30 vezes) durante um período de $24 \mathrm{~h}$ para que ocorresse a complexação dos íons metálicos pelos ligantes presentes no meio. Alíquotas desta amostra foram utilizadas na determinação, em triplicata, das concentrações totais dos íons metálicos $\mathrm{Cu}^{2+}, \mathrm{Pb}^{2+}, \mathrm{Cd}^{2+}$ e $\mathrm{Zn}^{2+}$. As alíquotas foram acidificadas com $\mathrm{HNO}_{3}(\mathrm{pH}<2,0)$ e digeridas em um reator fotoquímico ativado por micro-ondas, visando à eliminação da matéria orgânica presente na amostra. ${ }^{14}$ As concentrações totais dos íons metálicos foram determinadas simultaneamente empregando ASV com pulso diferencial pelo método da adição de padrão. O sistema voltamétrico utilizado foi um potenciostato EG\&PAR M394 acoplado a um sistema de eletrodos SMDE EG\&G PAR 303A com eletrodo de trabalho de gota pendente de mercúrio.
Tabela 1. Composição da amostra sintética de água natural

\begin{tabular}{cc}
\hline Composição & Concentração $\left(\mathrm{mg} \mathrm{L}^{-1}\right)$ \\
\hline $\mathrm{Cd}^{2+}$ & 0,0015 \\
$\mathrm{Cu}^{2+}$ & 0,0040 \\
$\mathrm{Fe}^{3+}$ & 0,025 \\
$\mathrm{~Pb}^{2+}$ & 0,0030 \\
$\mathrm{Zn}^{2+}$ & 0,020 \\
$\mathrm{AH}^{*}$ & 7,6 \\
$\mathrm{EDTA}^{2+}$ & 1,5 \\
$\mathrm{Cl}^{-}$ & 7,6 \\
$\mathrm{CO}_{3}{ }^{2-}$ & 54 \\
$\mathrm{SO}_{4}^{2-}$ & 11 \\
$\mathrm{Ca}^{2+}$ & 35 \\
$\mathrm{Mg}^{2+}$ & 4,0 \\
$\mathrm{Na}^{+}$ & 5,4 \\
$\mathrm{~K}^{+}$ & 8,4 \\
\hline
\end{tabular}

* AH: ácido húmico comercial (Aldrich)

Três dispositivos adquiridos da empresa DGT Research Ltd ${ }^{15}$ foram mantidos em agitação na amostra sintética durante $24 \mathrm{~h}$, tendo sido realizadas leituras periódicas do $\mathrm{pH}(7,0 \pm 0,1)$ e temperatura $\left(20,0 \pm 0,5^{\circ} \mathrm{C}\right)$. Ao final do experimento os dispositivos foram lavados com água Milli-Q e realizou-se a extração das espécies metálicas, acumuladas na resina, com 1,0 mL de $\mathrm{HNO}_{3}(1,0 \mathrm{~mol} \mathrm{~L}-1)$ por aproximadamente $12 \mathrm{~h}$, sob agitação. Os teores dos metais extraídos também foram determinados por ASV. As massas das espécies metálicas retidas na resina $(M)$ foram calculadas através Equação $1:^{9}$

$$
M=\frac{C_{e}\left(V_{g e l}+V_{\text {ácido }}\right)}{f_{e}}
$$

onde $C_{e}$ representa a concentração determinada do íon metálico eluído da resina $\left(\mathrm{g} \mathrm{cm}^{-3}\right), V_{\text {gel }}$ o volume do hidrogel-Chelex $\left(0,16 \mathrm{~cm}^{3}\right), V_{\text {ácido }}$ o volume do ácido usado na eluição $\left(1,0 \mathrm{~cm}^{3}\right)$ e $f_{e}$ o fator de eluição do íon metálico $(0,8) .{ }^{9}$ As concentrações das espécies metálicas presentes na forma lábil $\left(C^{\text {Lábil }}\right)$ determinadas por DGT foram calculadas de acordo com a Equação 2:

$$
C^{\text {Lábil }}=\frac{M \Delta g}{D A t}
$$

sendo $\Delta g$ a espessura da camada de difusão, que é a soma das espessuras do gel difusivo e da membrana de filtração $(0,094 \mathrm{~cm}), A$ representa a área superficial do hidrogel que fica exposta à solução $\left(3,14 \mathrm{~cm}^{2}\right), t$ o tempo de imersão do dispositivo (s) e $D$ o coeficiente de difusão do metal no hidrogel $\left(\mathrm{cm}^{2} \mathrm{~s}^{-1}\right)$. Os valores de $f_{e}, \Delta g, A$ e $D$ utilizados neste trabalho foram aqueles recomendados pela empresa que produz e comercializa os dispositivos DGT..$^{15}$

Seguindo recomendação do fabricante, foram feitos testes de adição e recuperação com cádmio para avaliar a eficiência do lote adquirido. O percentual de recuperação foi de $107 \pm 5 \%$, indicando que os dispositivos podem ser empregados com precisão e exatidão satisfatórias.

A análise de especiação dos metais no sistema modelo empregando ASV consistiu em realizar uma série de titulações da amostra, com força iônica ajustada em $0,1 \mathrm{~mol} \mathrm{~L}^{-1}$ com $\mathrm{KNO}_{3}$. Essas titulações foram individuais para cada elemento de interesse, com adições de diferentes concentrações do metal, seguidas de determinações voltamétricas após 20 min de tempo de contato. Os parâmetros instrumentais empregados na ASV com pulso diferencial foram: potencial de deposição de $-0,6 \mathrm{~V}(\mathrm{Ag} / \mathrm{AgCl})$ para $\mathrm{Cu}^{2+},-0,75 \mathrm{~V}$ para $\mathrm{Pb}^{2+},-0,9 \mathrm{~V}$ para $\mathrm{Cd}^{2+} \mathrm{e}-1,2 \mathrm{~V}$ para $\mathrm{Zn}^{2+}$ durante $300-600 \mathrm{~s}$, varredura de potencial 
de -1,2 a 0,15 V dependendo da espécie avaliada; tempo de equilíbrio de $10 \mathrm{~s}$; velocidade de varredura de $8 \mathrm{mv} \mathrm{s}^{-1}$ e amplitude de pulso de $50 \mathrm{mV}$. Detalhes sobre a obtenção das concentrações de metal lábil, assim como dos parâmetros de complexação, são apresentados no item Resultados e Discussão.

A modelagem da especiação foi realizada com o programa CHEAQS-Pro (CHemical Equilibria AQuatic Systems), versão P2008.1.0. ${ }^{16}$ Este programa encontra-se disponível em http://www. home.tiscalli.nl/cheaqs.

\section{RESULTADOS E DISCUSSÃO}

As concentrações totais dos íons metálicos determinadas são mostradas na Tabela 2. Comparando as concentrações adicionadas (Tabela 1) com aquelas determinadas, observa-se que as porcentagens de recuperação obtidas foram satisfatórias, variando de $80 \pm 10$ a 113 $\pm 6 \%$. Pode-se destacar que no sistema estudado as concentrações totais dos íons metálicos seguem a tendência encontrada comumente em amostras de águas naturais superficiais, ou seja, $\mathrm{Zn}^{2+}>\mathrm{Cu}^{2+}>$ $\mathrm{Pb}^{2+}>\mathrm{Cd}^{2+} .2,3$

Os resultados obtidos para as concentrações das espécies metálicas na forma lábil, através das três abordagens utilizadas, estão descritos na Tabela 2.

Tabela 2. Resultados obtidos nas determinações das concentrações totais e lábeis dos íons metálicos no sistema modelo

\begin{tabular}{lcccc}
\hline & \multicolumn{4}{c}{ Concentrações $\left(\mu \mathrm{g} \mathrm{L}^{-1}\right)$} \\
Metal & Total & DGT & AŚbil \\
& $1,20(0,04)$ & $<\mathrm{LQ}$ & $<\mathrm{LQ}$ & $0,005(0,002)$ \\
$\mathrm{Cd}$ & $4,50(0,02)$ & $0,59(0,01)$ & $0,71(0,02)$ & $0,01(0,02)$ \\
$\mathrm{Cu}$ & $3,25(0,02)$ & $<\mathrm{LQ}$ & $<\mathrm{LQ}$ & $0,007(0,002)$ \\
$\mathrm{Pb}$ & $20,50(0,03)$ & $0,530(0,004)$ & $0,72(0,01)$ & $2,40(0,02)$ \\
$\mathrm{Zn}$ &
\end{tabular}

LQ (limite de quantificação): 0,05 $\mu \mathrm{g} \mathrm{L} \mathrm{L}^{-1}$. Entre parênteses, estimativa do desvio padrão.

De acordo com os dados da Tabela 2, pelo método DGT foram obtidas baixas concentrações dos íons metálicos na forma lábil; 0,59 e $0,53 \mu \mathrm{g} \mathrm{L}^{-1}$ para cobre e zinco, respectivamente. Em termos percentuais, estas concentrações representam 13,1 e 2,6\% dos teores totais de $\mathrm{Cu}^{2+}$ e $\mathrm{Zn}^{2+}$. Na determinação por ASV dos íons cádmio e chumbo eluídos da resina Chelex não foram observados picos de redissolução. Consequentemente, as formas lábeis destes elementos não foram detectadas. Portanto, pode-se inferir que na amostra sintética os íons $\mathrm{Cu}, \mathrm{Pb}, \mathrm{Cd}$ e $\mathrm{Zn}$ se encontram majoritariamente complexados pelos ligantes orgânicos - ácido húmico e EDTA; em especial, pelo valor de $\mathrm{pH}(7,0)$ ser bastante favorável para propiciar a ocorrência do processo de complexação. ${ }^{5,17}$

Com relação à titulação voltamétrica, a Figura 1 mostra uma curva representativa deste procedimento. $\mathrm{O}$ gráfico relaciona a intensidade da corrente de pico do íon cobre com a soma das concentrações de cobre originalmente presente na amostra com as adicionadas durante a titulação. Na primeira parte da curva de titulação observam-se sinais de baixa intensidade de corrente, devido à complexação do cobre adicionado pelos ligantes disponíveis presentes na amostra. Quando ocorre a saturação dos sítios de complexação se nota uma mudança neste comportamento, com o surgimento de uma reta relacionando corrente e concentração com maior inclinação, dado que o íon metálico adicionado permanece na forma eletroativa (lábil). Com os dados obtidos na titulação foram determinadas as concentrações das espécies lábeis, pela razão entre a corrente e o coeficiente angular da equação da reta da parte linear da curva de titulação que apresenta uma maior inclinação. Pela diferença entre os teores do metal total e lábil foram calculadas as concentrações das espécies metálicas na forma complexada. ${ }^{4,18}$

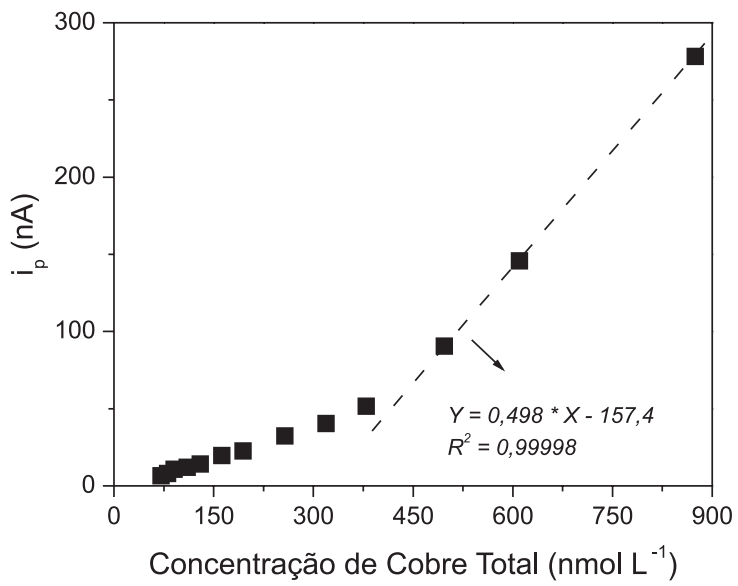

Figura 1. Curva de titulação da amostra com o íon cobre, obtida na análise de especiação por voltametria de redissolução anódica (ASV). Parâmetros instrumentais da análise por ASV: $E_{d e p}=-0,6 \mathrm{~V}(\mathrm{Ag} / \mathrm{AgCl}) ; t_{\text {dep }}$ $=420 \mathrm{~s} ; E_{i}=-0,6 \mathrm{~V} ; E_{f}=0,15 \mathrm{~V} ;$ amplitude do pulso $=50 \mathrm{mV}$; velocidade de varredura $=8 \mathrm{mV} \mathrm{s}^{-1}$

As características de complexação das amostras podem ser avaliadas através de tratamentos matemáticos dos dados obtidos na curva de titulação de acordo com a Equação $3:{ }^{18}$

$$
\frac{\left[M^{\prime}\right]}{[M L]}=\frac{1}{K_{M L}[L]}+\frac{\left[M^{\prime}\right]}{[L]}
$$

onde: $\left[M^{\prime}\right],[L]$ e $[M L]$ representam as concentrações do metal lábil, dos sítios disponíveis e do complexo metal-ligante, respectivamente. A constante de estabilidade condicional para o complexo $M L$ é representada por $K_{M L}$. Um exemplo da curva com a linearização dos dados da titulação é mostrado na Figura 2.

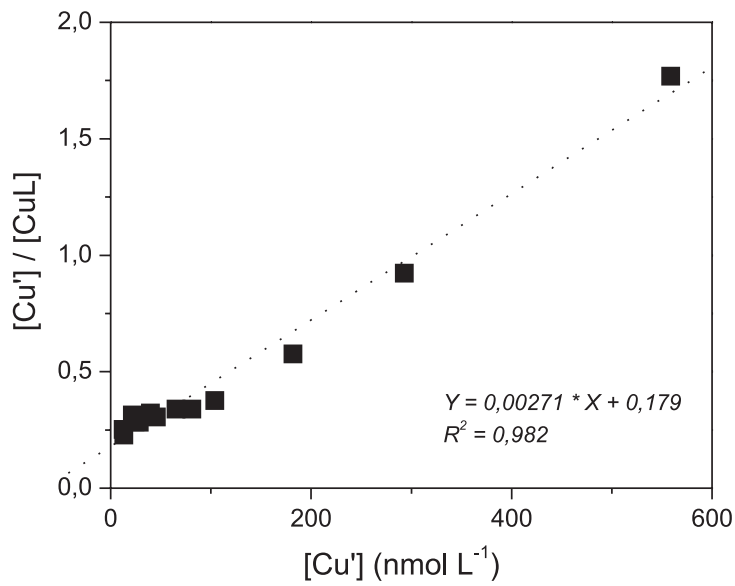

Figura 2. Gráfico de linearização dos dados obtidos na titulação voltamétrica da amostra sintética com o íon cobre, conforme Figura 1

As concentrações dos íons metálicos na forma lábil determinadas pelas titulações voltamétricas apresentam-se na mesma ordem de grandeza que os resultados obtidos por DGT (Tabela 2), apesar das diferenças nos protocolos e princípios de cada uma das abordagens. ${ }^{6,17}$ As titulações para os íons cádmio e chumbo foram caracterizadas pela 
ausência de picos voltamétricos referentes a estas espécies, o que sugere que esses elementos se encontram fortemente complexados na amostra sintética. Na maioria das situações, os complexos metálicos formados com ligantes orgânicos são inertes e não contribuem para a corrente voltamétrica. ${ }^{4,18}$

Os parâmetros de complexação $\left(L\right.$ e $\left.K_{M L}\right)$ foram calculados para os íons cobre e zinco empregando-se o modelo de linearização (Equação 3). Os valores do logaritmo da constante de estabilidade condicional, $\log K_{M L}$, determinados para CuL e ZnL foram 7,20 e 7,95, respectivamente. Os valores de $K_{M L}$ são da mesma ordem de grandeza para as interações dos dois elementos com os ligantes da amostra e indicam uma semelhança na estabilidade dos complexos formados entre os ligantes e os íons metálicos $\mathrm{Cu}^{2+} \mathrm{e} \mathrm{Zn}^{2+}$. Outro parâmetro avaliado foi a concentração de sítios disponíveis $(L)$ na amostra, cujos resultados

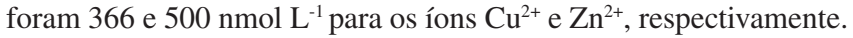
Assim, nota-se uma capacidade de complexação mais elevada para o zinco, fato que favorece a complexação desse elemento pelos ligantes presentes na amostra sintética. Os valores de $L$ e $K_{M L}$ obtidos estão em concordância com os valores encontrados em literatura, apesar da grande parte dos trabalhos serem voltados para amostras de águas naturais onde está presente uma variedade de ligantes que interagem com espécies metálicas, e nem sempre as condições experimentais utilizadas serem semelhantes. ${ }^{18}$

Pode-se afirmar, portanto, que a vantagem de se empregar a titulação voltamétrica em estudos de especiação está relacionada com a obtenção de informações sobre as características de complexação. Contudo, é importante mencionar que os valores obtidos para $K_{M L} \mathrm{e}$ $L$ dependem de inúmeros fatores, entre eles o método voltamétrico utilizado e a interpretação dos dados obtidos na titulação, uma vez que existem alguns modelos de linearização que podem levar em consideração a existência de uma ou mais classes de ligantes..$^{18}$

As concentrações de todas as espécies químicas da amostra foram calculadas com o CHEAQS, através do uso de balanços de massa e de carga. ${ }^{16}$ Neste programa está incluso o modelo WHAM - Windermere Humic Aqueous Model V, que deve ser utilizado em situações em que a especiação seja governada pela matéria orgânica, pois com esse modelo é possível prever a complexação das espécies metálicas pelas substâncias húmicas aquáticas, como ácido húmico e ácido fúlvico. ${ }^{17,19}$ As distribuições relativas dos íons $\mathrm{Cu}^{2+}, \mathrm{Cd}^{2+}, \mathrm{Pb}^{2+} \mathrm{e} \mathrm{Zn}^{2+}$ previstas na modelagem foram comparadas com os resultados obtidos pelas abordagens experimentais, conforme Figura 3. É importante ressaltar que as porcentagens de cada fração foram calculadas em relação aos teores totais determinados experimentalmente (Tabela 2).

No que diz respeito ao comportamento dos íons metálicos,

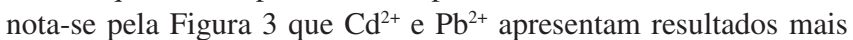

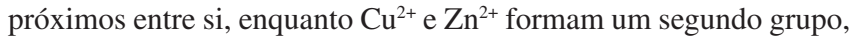
com comportamento diferenciado do primeiro.

Conforme mostra a Figura 3A, as frações complexadas dos íons cádmio e chumbo representam quase $100 \%$ da concentração total, uma vez que não foi possível realizar as determinações experimentais destes elementos na forma lábil, devido às concentrações serem extremamente baixas. Este resultado foi concordante com o CHEAQS, pois as formas lábeis estimadas representam menos que $0,5 \%$ dos teores totais. Assim, independentemente do método utilizado na análise de especiação, porcentagens similares das frações complexadas e lábeis para os íons $\mathrm{Cd}$ e $\mathrm{Pb}$ são observadas, o que demonstra a boa concordância entre os métodos avaliados.

Além de estimar as concentrações dos íons metálicos na forma lábil, o modelo CHEAQS também permitiu distinguir as frações complexadas entre os ligantes orgânicos EDTA e ácido húmico $(\mathrm{AH})$. O cádmio apresentou-se majoritariamente complexado pelo EDTA na amostra sintética, o que pode estar relacionado com a baixa afinidade das substâncias húmicas aquáticas por este metal, conforme descrito

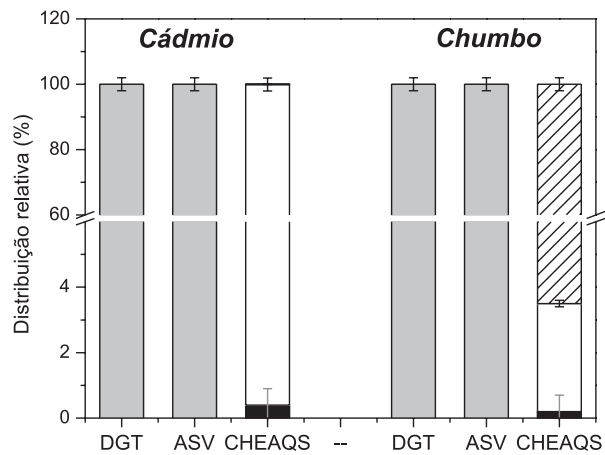

(A)

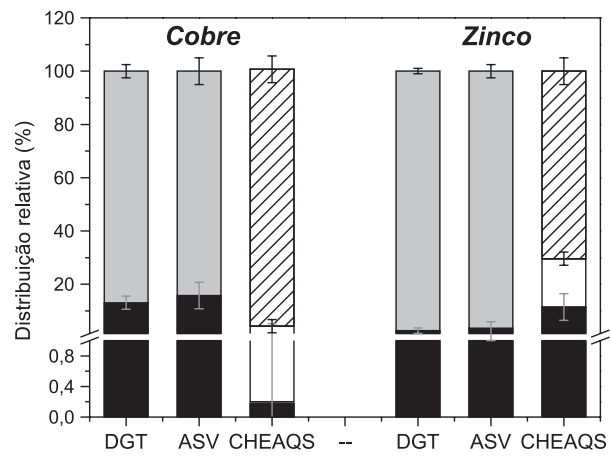

(B)

Figura 3. Comparação dos resultados obtidos na especiação dos íons $C d$ e $\mathrm{Pb}(\mathrm{A})$ e $\mathrm{Cu}$ e $\mathrm{Zn}(\mathrm{B})$ empregando difusão em filmes finos por gradiente de concentração (DGT), voltametria de redissolução anódica (ASV) e programa CHEAQS. Metal lábil em preto, metal complexado em cinza, metal-AH em listras e metal-EDTA em branco

na literatura. ${ }^{18,20}$ Abate e Masini avaliaram a complexação de alguns metais pelo $\mathrm{AH}$ (Aldrich) e interações pouco pronunciadas do cádmio com este agente complexante foram verificadas. ${ }^{18}$

Nos cálculos referentes ao chumbo observa-se que a fração complexada pelo ácido húmico foi bastante superior em relação às outras frações. Este comportamento está concordante com a literatura, que indica que materiais húmicos apresentam elevada afinidade por este íon metálico. ${ }^{2,7,18}$ Alonso e colaboradores avaliaram a especiação de metais em águas superficiais do Rio Guadiamar, na Espanha, e verificaram que o chumbo se encontrava em formas pouco disponíveis, devido à presença da matéria orgânica dissolvida. ${ }^{20}$

Em vários trabalhos foi evidenciada a influência da matéria orgânica, principalmente as substâncias húmicas, na dinâmica e na especiação de metais em ambientes aquáticos. Em muitos casos, devido à predominância da complexação orgânica das espécies metálicas, ocorre uma diminuição da biodisponibilidade desses elementos, uma vez que estes complexos não são facilmente transportados através da membrana celular de organismos vivos. ${ }^{18-20}$

Com relação à distribuição dos íons metálicos $\mathrm{Cu}$ e Zn no sistema estudado, observa-se na Figura 3B que as frações lábeis foram expressivamente inferiores às frações complexadas, devido à presença de ligantes orgânicos que formam complexos estáveis com ambas as espécies metálicas. ${ }^{20}$ De modo geral, observa-se a ocorrência de porcentagens mais elevadas da forma complexada para o zinco, em comparação com o cobre, confirmando os resultados obtidos por voltametria, que mostraram uma maior capacidade de complexação da amostra frente ao $\mathrm{Zn}$.

$\mathrm{Na}$ distinção entre os complexos formados com EDTA e ácido húmico para os íons metálicos $\mathrm{Cu}$ e $\mathrm{Zn}$ realizada com o emprego do modelo, verifica-se que ambos os metais formaram complexos 
preferencialmente com o AH. A formação de complexos fortes entre substâncias húmicas e estes elementos, especialmente $\mathrm{Cu}$, está bem descrita na literatura. ${ }^{6,10,18,20}$

No que diz respeito à comparação entre ASV e DGT, a Figura 3 evidencia que os resultados obtidos não apresentam diferenças significativas, embora estes métodos sejam diferentes entre si tanto nos procedimentos quanto nos princípios. ${ }^{9,17}$ Vale destacar que apesar das porcentagens médias de metal lábil não serem diferentes estatisticamente, as concentrações obtidas pela titulação voltamétrica foram um pouco superiores, sistematicamente, em relação àquelas obtidas através do DGT. Estes resultados podem estar relacionados com as diferentes formas de distinção e detecção das espécies características da ASV e DGT, uma vez que ambos os métodos são dependentes da dissociação dos complexos metálicos, que pode ocorrer durante a realização das medidas. Assim, são medidos os íons metálicos livres, complexos inorgânicos e alguns complexos orgânicos dependendo de fatores como cinética e estabilidade dos complexos, assim como das características de cada método utilizado, entre outros. ${ }^{9,21}$ Como foi obtida uma menor fração dos metais lábeis pelo método DGT, o resultado pode indicar que foram determinadas concentrações menores dos complexos metálicos formados com ligantes orgânicos reversíveis, ou seja, aqueles complexos capazes de sofrerem dissociação. Este comportamento pode sugerir que o hidrogel retarda a difusão dos complexos orgânicos mais reversíveis, uma vez que este material apresenta uma estrutura polimérica com poros de tamanhos específicos que controlam o transporte difusional de espécies no sistema DGT. De fato, Zhang e Davison relataram que os complexos metálicos formados com substâncias húmicas difundem mais lentamente que os íons metálicos lábeis pelo hidrogel do DGT. ${ }^{17}$

Na medida empregando DGT é estabelecido um gradiente de concentração e a massa da espécie metálica acumulada na resina Chelex deve-se ao fluxo das espécies metálicas lábeis que se difundem, durante um determinado tempo, no hidrogel (camada de difusão). O fluxo $(J)$ é dado pela $1^{\text {a }}$ Lei de Difusão de Fick, descrita na Equação 4.

$$
J=\frac{d C}{d x}=\frac{D C}{\Delta g}
$$

onde $C, D$ e $\Delta g$ representam a concentração do íon metálico, o coeficiente de difusão e a espessura da camada de difusão, respectivamente. ${ }^{9}$

Portanto, a medida da labilidade de íons metálicos pelo método DGT depende de parâmetros tais como a difusibilidade das espécies metálicas, o tempo de residência das espécies lábeis na camada de difusão e, principalmente, da espessura da camada de difusão $0^{9,17,21}$, cujo valor é aproximadamente $0,1 \mathrm{~mm}$. $^{4,17,21}$

Diferentemente do DGT, a voltametria baseia-se nos fenômenos que ocorrem entre o eletrodo de trabalho e a fina camada de solução adjacente a essa superfície. Na ASV na etapa de pré-concentração os metais lábeis alcançam o eletrodo por convecção e difusão. Na etapa seguinte, de redissolução, as espécies reduzidas no eletrodo são reoxidadas, sendo este processo governado por difusão. Dessa maneira, a corrente medida é proporcional ao fluxo das espécies lábeis, conforme a Equação $5 .^{17}$

$$
J=\frac{D_{1} C_{1}}{\delta}+\frac{D_{2} C_{2}}{\delta}+\frac{D_{3} C_{3}}{\delta}+\ldots
$$

onde $D, C$ e $\delta$ são os coeficientes de difusão das espécies metálicas individuais, as concentrações dessas espécies e a espessura da camada de difusão, respectivamente. A labilidade dos íons metálicos determinada por ASV também depende da espessura da camada de difusão, que é estimada em $10 \mu \mathrm{m}$, aproximadamente. ${ }^{17}$
Comparando-se DGT e ASV, observa-se que a espessura da camada de difusão no caso do método voltamétrico é cerca de 10 vezes inferior àquela do DGT. Pelas Equações 4 e 5 verifica-se que o fluxo e a concentração das espécies metálicas lábeis são inversamente proporcionais à espessura da camada de difusão. Portanto, maiores teores de metal lábil podem ser obtidos por voltametria, devido à menor espessura da camada de difusão. Nossos resultados corroboram valores obtidos em outros estudos, entre eles os de Scally et al..$^{21}$ e Davison e Zhang. ${ }^{9}$ Estes autores também relatam que existe uma relação inversamente proporcional entre a massa acumulada nos dispositivos DGT e a espessura da camada de difusão.

Desta forma, pode-se concluir que os resultados obtidos na análise de especiação dos íons metálicos $\mathrm{Cu}, \mathrm{Cd}, \mathrm{Pb}$ e $\mathrm{Zn}$, neste estudo, empregando distintas abordagens, podem ser considerados concordantes e satisfatórios. Também é possível afirmar que o uso do CHEAQS possibilitou prever a especiação dos metais de maneira eficiente, apesar da previsão para o cobre lábil ter sido inferior aos resultados experimentais (Figura 3B). Esta situação pode estar relacionada com o uso do modelo WHAM-V, que em muitos casos superestima a complexação do cobre por substâncias húmicas. Guthrie e coautores ${ }^{19}$ também relatam uma pobre concordância entre dados experimentais e previstos pelo modelo WHAM para o íon $\mathrm{Cu}$ e concluem que a principal limitação desse modelo decorre da dificuldade de se estabelecer um valor confiável para a concentração de carbono orgânico, o qual está relacionado com a presença de complexantes naturais como grupos carboxílicos e fenólicos, ativamente envolvidos nas interações entre substâncias húmicas e espécies metálicas. Vários trabalhos demonstram a dificuldade de se relacionar dados experimentais com dados da modelagem de especiação de metais em ambientes aquáticos, ${ }^{6,8}$ dadas as limitações inerentes aos programas de modelagem, especialmente por serem sensíveis à qualidade da sua base de dados, por requererem o conhecimento completo da composição das amostras e por não contemplarem aspectos cinéticos, entre outros.

Assim, o estudo realizado em sistema modelo, sob condições controladas, possibilitou a interpretação dos dados obtidos em análises de especiação dos íons $\mathrm{Cu}^{2+}, \mathrm{Cd}^{2+}, \mathrm{Pb}^{2+}$ e $\mathrm{Zn}^{2+}$ com maior confiança, sendo esta situação ideal na avaliação comparativa entre diferentes protocolos analíticos.

\section{CONCLUSÕES}

Em virtude dos aspectos mencionados neste trabalho, pode-se concluir que os experimentos conduzidos em sistema modelo proporcionaram uma avaliação satisfatória da labilidade de espécies metálicas empregando ASV, CHEAQS e DGT, dado que foram obtidos resultados concordantes. A escolha da melhor abordagem a ser utilizada em estudos de especiação depende do tipo de informação que se deseja obter, além dos recursos logísticos e operacionais disponíveis, porém qualquer método utilizado apresenta vantagens e limitações. Neste sentido, pode-se concluir que o método DGT apresenta inúmeras vantagens frente aos métodos ASV e modelagem, tais como facilidade de uso e implantação em análises de rotina, facilidade de realizar medidas in situ, rápida obtenção de resultados e, ainda, por fornecer informações que representam valores médios para o período de sua utilização em ambientes aquáticos. Porém, o emprego do DGT requer o conhecimento prévio sobre a difusibilidade do analito no hidrogel e da capacidade de retenção da resina Chelex. A ASV proporciona como atrativo a possibilidade de se obter dados sobre as características de complexação das amostras. Contudo, adulterações da amostra podem ocorrer por se tratar de um procedimento moroso e ex situ.

A capacidade de previsão da especiação de metais sem medidas experimentais é a grande utilidade dos modelos computacionais, 
porém o êxito deste tipo de abordagem é difícil devido à heterogeneidade dos ambientes aquáticos, pela dependência do conhecimento completo da amostra e da base de dados do programa. Portanto, considerando-se a importância da análise de especiação de metais em processos ambientais e biológicos, o conhecimento das limitações de cada protocolo analítico é uma questão de extrema relevância.

\section{AGRADECIMENTOS}

Ao CNPq e CAPES pelo suporte financeiro e bolsa de estudos de V. E. Anjos.

\section{REFERÊNCIAS}

1. Slaveykova, V. I.; Karadjova, I. B.; Karadjova, M.; Tsalev, D. L.; Environ. Sci. Technol. 2009, 43, 1798; Tonello, P. S.; Rosa, A. H.; Abreu, C. H. J.; Menegário, A. A.; Anal. Chim. Acta 2007, 598, 162.

2. Stumm, W.; Morgan, J. J.; Aquatic Chemistry, $3^{\text {rd }}$ ed., Wiley: New York, 1996; Balistrieri, L. S.; Blank, R. G.; Appl. Geochem. 2008, 23, 3355; Bowles, K. C.; Apte, S. C.; Batley, G. E.; Hales, L. T.; Rogers, N. J.; Anal. Chim. Acta 2006, 558, 237.

3. Odzak, N.; Kistker, D.; Xue, H.; Sigg, L.; Aquat. Sci. 2002, 64, 292; Florence, T. M.; Talanta 1982, 29, 345; Allen, H. E.; Hansen, D. J.; Water Environ. Res. 1996, 68, 42; Sodré, F. F.; Grassi, M. T.; Water, Air, Soil Pollut. 2007, 178, 103; Chakraborty, P.; Zhao, J.; Chakrabarti, C. L.; Anal. Chim. Acta 2009, 636, 70.

4. Batley, G. E.; Apte, S. C.; Stauber, J. L.; Aust. J. Chem. 2004, 57, 903; Pesavento, M.; Alberti, G.; Biesuz, R.; Anal. Chim. Acta 2009, 631,129; Florence, T. M.; Analyst 1986, 111, 489.

5. Sigg, L.; Black, F.; Buffle, J.; Cao, J.; Cleven, B.; Davison, W.; Galceran, J.; Gunkel, P.; Kalis, E.; Kistler, D.; Martin, M.; Noel, S.; Nur, Y.; Odzak, N.; Puy, J.; van Riemsdijk, W.; Temminghoff, E.; Waeber, M. L. T.; Toepperwien, S.; Town, R. M.; Unsworth, E.; Warnken, K. W.; Weng, L.; Xue, H.; Zhang, H.; Environ. Sci. Technol. 2006, 40, 1934; van Leeuwen, H. P.; Town, R. M.; Buffle, J.; Cleven, R. F. M. J.; Davison, W.; Puy, J.; van Riemsdijk, W. H.; Sigg, L.; Environ. Sci. Technol. 2005, 39, 8545.

6. Meylan, S.; Odzak, N.; Behara, R.; Sigg, L.; Anal. Chim. Acta 2004, 510, 91; Dunn, R. J. K.; Teasdale, P. R.; Warnken, J.; Jordan, M. A.; Arthur, J. M.; Environ. Pollut. 2007, 148, 213.

7. Meylan, S.; Behra, R.; Sigg, L.; Environ. Sci. Technol. 2003, 37, 5204; Kozelka, P. B.; Bruland, K. W.; Mar. Chem. 1998, 60, 267.

8. Unsworth, E. R.; Warnken, K. W.; Zhang, H.; Davison, W.; Black, F.; Buffle, J.; Cao, J.; Cleven, R.; Galceran, J.; Gunkel, P.; Kalis, E.; Kistler, D.; van Leeuwen, H. P.; Martin, M.; Noel, S.; Nur, Y.; Odzak, N.; Puy, J.; van Riemsdijk, W.; Sigg, L.; Temminghoff, E.; Waber-Tercier, M. L.; Toepperwien, S.; Town, R. W.; Wheng, L.; Xue, H.; Environ. Sci. Technol. 2006, 40, 1942.
9. Davison, W.; Zhang, H.; Nature 1995, 367, 546; Zhang, H.; Davison, W.; Anal. Chem. 1995, 67, 3391; Kersanach, M. W.; Andrade, C. F. F.; Zhang, H.; Milani, M. R.; Niencheski, L. F. H.; J. Braz. Chem. Soc. 2009, 20, 333; Zhang, H., Davison, W.; Pure Appl. Chem. 2001, 73, 9; Zhang, H.; Environ. Sci. Technol. 2004, 38, 1421.

10. Gimpel, J.; Zhang, H.; Davison, W.; Edwards, A. C.; Environ. Sci. Technol. 2003, 37, 138; Senn, D. B.; Griscom, S. B.; Lewis, C. G.; Galvin, J. P.; Chang, M. W.; Shine, J. P.; Environ. Sci. Technol. 2004, 38, 3381; Garmo, O. A.; Davison, W.; Zhang, H.; Anal. Chem. 2008, 80, 9229.

11. Unsworth, E. R.; Zhang, H.; Davison, W.; Environ. Sci. Technol. 2005, 39, 624; Twiss, M. R.; Moffett, J. W.; Environ. Sci. Technol. 2002, 36, 1061.

12. Campos, M. L. A.; Bendo, A.; Viel, F. C.; Quim. Nova 2002, 25, 808.

13. Campbell, P. G. C. Em Metal speciation and bioavailability in aquatic system; Tessier, A.; Turner, D. R., eds.; Wiley: New York, 1995.

14. Sodré, F. F.; Zamora, P. G. P.; Grassi, M. T.; Quim. Nova 2004, 27, 695. 15. http://www.dgtresearch.com, acessada em Abril 2008.

16. Verweij, W.; CHEAQS PRO 2008.1.0, A program for calculating CHemical Equilibria in AQuatic Systems; Netherlands, 1999-2008.

17. Zhang, H.; Davison, W.; Anal. Chem. 2000, 72, 4447.

18. van den Berg, C. M. G.; Kramer, J. R.; Anal. Chim. Acta 1979,106, 113; Ruzic, I.; Anal. Chim. Acta 1982, 140, 99; Scheffer, E, W.; Sodré, F. F.; Grassi, M. T.; Quim. Nova 2007, 30, 332; Abate, G.; Masini, J. C.; Quim. Nova 1999, 22, 661.

19. Tipping, E.; Comput. Geosci. 1994, 20, 973; Bryan, S.; Tipping, E.; Taylor, J. H.; Environ. Geochem. Health 1999, 21, 305; Guthrie, J. W.; Hassan, N. M.; Salam, M. S. A.; Fasfous, I. I.; Murimboh, C. A.; Murimboh, J.; Chakrabarti, C. L.; Grégoire, D. C.; Anal. Chim. Acta 2005, 528, 205.

20. Rosa, A. H.; Goveia, D.; Bellin, I. C.; Tonello, P. S.; Antunes, M. L. P.; Dias Filho, N. L.; Rodrigues Filho, U. P.; Quim. Nova 2007, 30, 59; Bruland, K. W.; Rue, E. L.; Donat, J. R.; Skrabal, S. A.; Moffett, J. W.; Anal. Chim. Acta 2000, 405, 99; Donat, J. R.; Anal. Chim. Acta 1994, 284, 547; Turoczy, N. J.; Sherwood, J. E.; Anal. Chim. Acta 1997, 354, 15; Alonso, E.; Santos, A.; Callejón, M.; Jiménez, J. C.; Chemosphere 2004, $56,561$.

21. Warnken, K. W.; Zhang, H.; Davison, W.; Anal. Chem. 2005, 77, 5440; Sangri, M. R.; Halstead, M. J.; Hunter, K. A.; Anal. Chim. Acta 2002, 456, 241; Scally, S.; Davison, W.; Zhang, H.; Environ. Sci. Technol. 2003, 37, 1379. 